\title{
Inter-annual recurrence of archaeal assemblages in the coastal NW Mediterranean Sea (Blanes Bay Microbial Observatory)
}

\author{
Pierre E. Galand, a,c,d, ${ }^{*}$ Carmen Gutiérrez-Provecho, ${ }^{a}$ Ramon Massana,b Josep M. Gasol,b \\ and Emilio O. Casamayor ${ }^{a}$ \\ a Department of Continental Ecology-Limnology, Centre d'Estudis Avançats de Blanes (CSIC), Blanes, Spain \\ b Departament de Biologia Marina i Oceanografia, Institut de Ciències del Mar (CSIC), Barcelona, Spain \\ c Université Pierre et Marie Curie (UPMC) Paris 06, Observatoire Océanologique, Banyuls-sur-Mer, France \\ dCentre National de la Recherche Scientifique (CNRS), FRE 3350, Laboratoire d'écogéochimie des environnements benthiques \\ (LECOB), Observatoire Océanologique, Banyuls-sur-Mer, France
}

\begin{abstract}
We report a long-term (i.e., 4.5 consecutive yr) monitoring of surface marine archaeal assemblages of the coastal Mediterranean Sea using quantitative polymerase chain reaction against specific phylogenetic and functional genes, and, for some specific samples, clone libraries of the $16 \mathrm{~S}$ ribosomal ribonucleic acid gene. Archaea had a marked seasonal periodicity, with recurrent peaks of abundance in December and January and very low occurrence during summer, parallel to temporal changes in community composition. Group II.b Euryarchaeota sequences were mostly present during winter when water was nutrient-enriched, and phytoplankton were abundant. Group II.a sequences were, in turn, more abundant during summer when the water column is stratified, and nutrient concentrations and phytoplankton stocks were lower. Group I Crenarchaeota abundance was highest during winter and significantly correlated with that of archaeal ammonia monooxygenase $($ amo $A$ ) gene copies and nitrite concentrations, suggesting that Group I Crenarchaeota were ammonia oxidizers. The periodicity of archaeal assemblages matched the strong and predictable seasonality of the surface-water conditions in the northwestern Mediterranean Sea, and suggests a low degree of functional redundancy between archaeal groups. The distinct seasonal dynamics for Group II.a and II.b Euryarchaeota, and their close association with major ecosystem processes, indicate that they may play an important but as yet largely unknown role in the ocean.
\end{abstract}

Marine archaea are important in terms of metabolic activity and abundance (Karner et al. 2001; Wuchter et al. 2006). The most common phyla in the ocean are Crenarchaeota Group I and Euryarchaeota Group II (Massana et al. 2000), while Euryarchaeota Group III is rare and restricted to deep waters (Galand et al. 2009a). The physiology and ecology of Group II Euryarchaeota remain poorly understood. Nevertheless, the genomes of some bathypelagic Group II Euryarchaeota enriched in putative anaerobic respiration components, suggest that some may have anaerobic pathways in the deep sea (Martin-Cuadrado et al. 2008). And conversely, in surface waters some phylotypes of Group II Euryarchaeota (a group often retrieved in the photic zone [Massana et al. 2000]), possess a gene coding for light-capturing proteorhodopsins (Frigaard et al. 2006), which may indicate that some Group II Euryarchaeota could use light as an additional energy source.

In contrast to the poor understanding of Euryarchaeota's role in the ocean, intense research on Group I Crenarchaeota has led to key discoveries linking the group to carbon and nitrogen biogeochemical cycling. Several lines of evidence indicate that they are fixing $\mathrm{CO}_{2}$ and that chemoautotrophy is the dominant archaeal metabolism in deep waters (Ingalls et al. 2006). The cultivation of Nitrosopumilus maritimus, an ammonia-oxidizing autotro-

\footnotetext{
*Corresponding author: pierre.galand@obs-banyuls.fr
}

phic Crenarchaeota (Könneke et al. 2005), and the widespread occurrence of the archaeal ammonia monooxygenase gene (amo A) in the sea (Francis et al. 2005) have further highlighted their importance in the nitrogen cycle. Even though Crenarchaeota may be more important than marine bacteria driving the oxidation of ammonia to nitrite (Wuchter et al. 2006), new data indicate that not all Crenarchaeota are ammonia-oxidizers living autotrophically (Agogue et al. 2008; Pouliot et al. 2009). Evidence that Crenarchaeota take up organic compounds such as amino acids (Ouverney and Fuhrman 2000) suggests that some Crenarchaeota could be heterotrophs or mixotrophs. Several studies have shown that Crenarchaeota tend to be more abundant in surface waters during winter under many different oceanic regimes (Santa Barbara Channel in California, waters off Antarctic Peninsula, and the North Sea [Massana et al. 1997; Murray et al. 1998; Herfort et al. 2007]). In warmer oligotrophic marine waters such as those of the Mediterranean Sea, Crenarchaeota have been detected in both surface and deep waters (Garcia-Martinez and Rodriguez-Valera 2000). Their seasonal variability remains, however, poorly understood because they were sometimes more frequent in surface winter waters (Winter et al. 2009) but not always (Alonso-Sáez et al. 2007).

Attempts to unveil the role and diversity of marine microbes are challenged by the spatial and temporal intricacy of the marine environment. One solution to deal with such complexity is to investigate many oceanic regimes, representing different environmental conditions, 
through both large-scale projects like the Global Ocean Survey, or international cooperative efforts such as the International Census of Marine Microbes. Those extensive global approaches can, however, miss the temporal component of microbial dynamics. A complementary strategy is to monitor microbes and ancillary environmental parameters at only one location, but over a long period of time, through the implementation of microbial observatories. Such marine microbial observatories have demonstrated reoccurring and predictable patterns in bacterial communities (Fuhrman et al. 2006) and have unveiled bacterial seasonal dynamics (Alonso-Sáez et al. 2007).

The long-term $(>2 \mathrm{yr})$ dynamics of marine archaea remain unexplored despite their important links with the oceanic carbon and nitrogen cycles. Here, we monitored archaeal communities in monthly intervals from January 2003 to May 2007 at the Blanes Bay Microbial Observatory, a site representative of the coastal northwest Mediterranean Sea, and characterized by a marked seasonality with high irradiance, and relatively warm, salty, and nutrient-poor waters (Duarte et al. 1999). We tested the hypothesis that archaeal groups show recurrent and predictable seasonal patterns by quantifying whole Archaea, Group II Euryarchaeota, Group I Crenarchaeota, and the functional amo $A$ genes by quantitative polymerase chain reaction (qPCR). We also described phylotype dynamics through archaeal 16S ribosomal ribonucleic acid (rRNA) gene cloning and sequencing at selected periods.

\section{Methods}

Sampling and environmental parameters - Surface seawater samples $(0.5 \mathrm{~m})$ were collected monthly at the Blanes Bay Microbial Observatory $\left(41^{\circ} 40^{\prime} \mathrm{N}, 2^{\circ} 48^{\prime} \mathrm{E} ; 20 \mathrm{~m}\right.$ depth) in the northwestern Mediterranean from January 2003 to May 2007 (53 samples). Water was filtered through a 200$\mu \mathrm{m}$-mesh net and kept in 25-liter polycarbonate carboys in the dark until being processed in the laboratory (within $1.5 \mathrm{~h})$.

Methods for determination of surface-water temperature, chlorophyll a (Chl a) concentration, dissolved inorganic nutrient concentrations, and heterotrophic prokaryotes numbers were described earlier (Alonso-Sáez et al. 2008).

Deoxyribonucleic acid (DNA) extraction, PCR amplification, and cloning-Microbial biomass was collected by sequentially filtering 8 liters of seawater through a $3-\mu \mathrm{m}$ pore-size polycarbonate filter (Poretics) and a $0.2-\mu \mathrm{m}$ Sterivex filter (Durapore, Millipore). Nucleic acids were extracted as described earlier (Massana et al. 1997). The concentration of DNA was measured by a NanoDrop. Archaeal 16S rRNA genes were amplified with primers $109 \mathrm{~F}$ and $915 \mathrm{R}$ as previously described (Galand et al. $2009 b$ ). PCR products were purified with Qiaquick PCR Purification Kit (Qiagen) and cloned with TA cloning kit (Invitrogen). In total we constructed eight archaeal clone libraries covering year 2003. We chose to clone samples from year 2003 to allow comparison with bacterial sequences published earlier (Alonso-Sáez et al. 2007). For each library, clones containing inserts were randomly chosen and directly sequenced using the vectors' $\mathrm{T} 7 \mathrm{p}$ primer. Putative chimeras were checked by using basic local alignment search tool (BLAST) with sequence segments separately. The 16S rRNA sequence data have been archived in the GenBank database under accession Nos. GQ387680-GQ387925.

Quantitative PCR ( $q P C R$ )-The number of whole archaeal and Group I crenarchaeal-specific 16S rRNA genes, as well as archaeal amo $A$ gene copy numbers were quantified for all samples in triplicate following conditions, reagents, and efficiencies described earlier (Galand et al. 2009b). For Group II euryarchaeal 16S rRNA gene copies we used primers GII-554-f (GTC GMT TTT ATT GGG CCT AA; Massana et al. 1997) and Eury806-r (CAC AGC GTT TAC ACC TAG; Teira et al. 2004) with similar qPCR conditions but with $59^{\circ} \mathrm{C}$ annealing temperature. All reactions were performed with $2 \mathrm{ng}$ of template on a DNA Engine Thermal Cycler with Chromo 4 Real-Time Detector (Bio-Rad) using the fluorescent dye SYBRGreen I. Standards obtained from purified PCR products of clones containing the targeted gene were subjected to qPCR along with the samples (Galand et al. 2009b). Primer specificity was confirmed by melting curve analyses.

The specificity of the qPCR primers was also estimated with the OligoCheck function within the Primerose software package (Ashelford et al. 2002) against a database containing the Blanes Bay sequences obtained by cloning. The qPCR primers generally matched well the Blanes Bay sequences but there were a few exceptions. The forward qPCR primer for Group I Crenarchaeota had two mismatches with all sequences belonging to cluster B (see Web Appendix, www.aslo.org/lo/toc/vol_55/issue_5/2117a. pdf). The reverse primer for Group II Euryarchaeota had one mismatch to all sequences from the VIM2-36 clade of Group II.a Cluster L (see Web Appendix). Because we were interested in monitoring dynamics rather than quantifying absolute copy numbers, and because qPCR primers may miss some archaeal targets, we decided to present the quantification results as relative to a yearly averaged value, rather than absolute copy numbers, as in Hansman et al. (2009). qPCR results were normalized for each year separately to avoid possible quantification bias associated with different length of DNA storage. DNA levels decline substantially over time (Sozzi et al. 2005) and the samples we stored for $>5 \mathrm{yr}$ may yield lower copy numbers than the ones stored for $1 \mathrm{yr}$ only. Results are, thus, expressed as a relative abundance of gene copies per ng of total DNA normalized to yearly averaged gene abundances (normalized average $=1$ ).

Phylogenetic analysis, diversity calculations, and statistic analysis - Sequences were compared with those in the GenBank database using the BLAST server at the National Center for Biotechnology Information. The $\sim 800$-base-pair sequences were aligned using the multiplesequence comparison by log-expectation (MUSCLE) pack- 

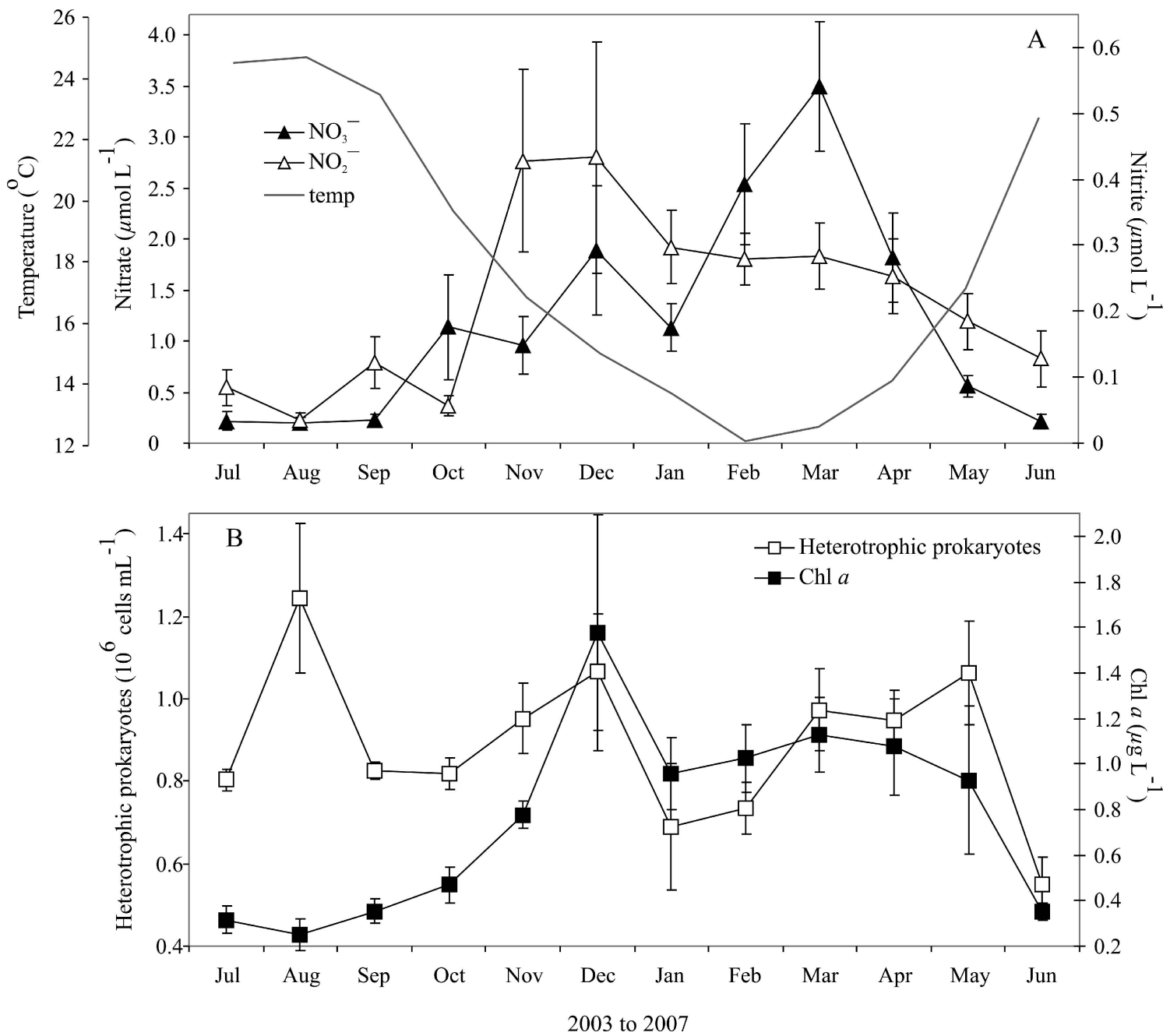

Fig. 1. Monthly average measurements $( \pm \mathrm{SE})$ of $(\mathrm{A})$ Nitrate $\left(\mathrm{NO}_{3}^{-}\right)$, nitrite $\left(\mathrm{NO}_{2}^{-}\right)$, and temperature, and (B) bacteria and Chl $a$ concentrations through the period January 2003-May 2007 at the Blanes Bay Microbial Observatory.

age (Edgar 2004) and manually checked. Phylogenetic analyses were completed with the program PHYLIP version 3.68 (Felsenstein 2008). DNADIST was used to calculate genetic distances with Kimura-2 model with 1000 data sets obtained by bootstrapping, and the distance tree was estimated with FITCH.

Sequences were grouped in operational taxonomic units (OTU) with the program DOTUR (Schloss and Handelsman 2005) through a Jukes-Cantor-corrected distance matrix obtained using the DNADIST program from PHYLIP, and rarefaction curves were calculated at a 97\% identity cut off.

Correlations between the relative abundances of Archaea, Group II Euryarchaeaota, Group I Crenarchaeota, amo $A$, and environmental factors (temperature, salinity, Chl $a$, nitrate, nitrite, ammonium, phosphate, and silicate) were examined with the nonparametric Spearman's coefficients $(r)$ because some variables did not comply with normality. Correlations were tested for significance at $\alpha=$ 0.05 using a $t$-test as implemented in the program PAST, version 1.91 (Hammer et al. 2001).

\section{Results}

Physico-chemical and biological parameters-Average monthly values measured throughout the $4.5-\mathrm{yr}$ survey showed that surface-water temperatures had clear seasonality, with values averaging $22-24^{\circ} \mathrm{C}$ in summer (Jun to Sep) and $12-14^{\circ} \mathrm{C}$ in winter (Dec to Mar; Fig. 1A). Nitrate and nitrite also showed seasonality, with higher concentrations in winter than in summer. The highest nitrite concentrations were found between November and January, whereas nitrate peaked along February-March (Fig. 1A). Ammonium concentrations did not show any seasonal trend (data not shown).

Chl $a$ concentrations were on average five times higher in winter and early spring than in summer (Fig. 1B), and were strongly correlated to nitrate (Spearman correlation, $n=$ $53, r=0.65, p<0.001)$ and nitrite $(n=53, r=0.49, p=$ $0.01)$ concentrations. Heterotrophic prokaryotes concentrations ranged between $0.5 \times 10^{6}$ cells mL $\mathrm{mL}^{-1}$ and $1.5 \times 10^{6}$ cells $\mathrm{mL}^{-1}$ and followed the Chl $a$ dynamic during winter and spring. 


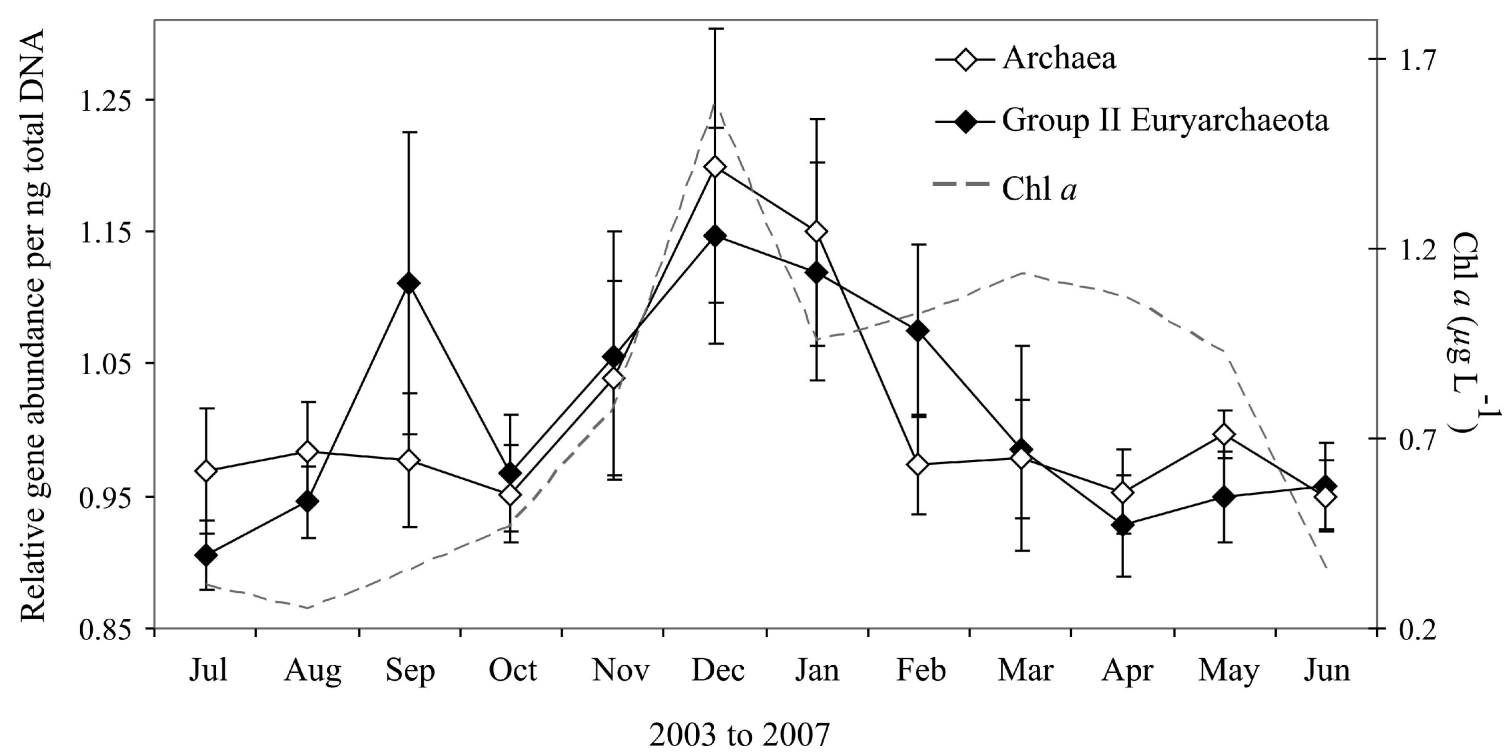

Fig. 2. Average seasonal abundance $( \pm \mathrm{SE})$ of Archaeal and Euryarchaeal $16 \mathrm{~S}$ rRNA gene. Gene copy numbers were quantified by qPCR through the period January 2003-May 2007 at the Blanes Bay Microbial Observatory. Data are normalized to yearly average values $(=1)$.

Long-term archaeal and amoA gene dynamics-The inter-annual qPCR quantification of the general archaeal $16 \mathrm{~S}$ rRNA gene showed that archaeal relative abundance was higher in winter than in summer, with peaks detected in early winter (Dec to Jan; Fig. 2). Concentrations of Archaea were significantly correlated to silicate only (negative correlation, $n=53, r=-0.30, p=0.03$ ) among all the environmental parameters we tested (i.e., surface temperature, salinity, Chl $a$, nitrate, nitrite, ammonium, phosphate, silicate). The archaeal peak matched, however, the early winter Chl $a$ abundance, but did not follow the later Chl $a$ dynamics corresponding to the main late-winter phytoplankton bloom (Fig. 2).
Specific qPCR quantifications were done for Group II Euryarchaeota (Fig. 2) and Group I Crenarchaeota (Fig. 3). Euryarchaeota abundance was correlated to the abundance of total Archaea $(n=53, r=0.34, p=0.01)$ and followed a similar seasonal dynamic, with the highest abundance during winter (Nov to Mar) and a peak in December. The high abundance in September (Fig. 2) was due to an episodic peak of Group II Euryarchaeota in 2005 and was not considered as representative of the long-term euryarchaeal dynamics. Group I Crenarchaeota were more abundant during late autumn-early winter (Nov to Jan; Fig. 3) and were strongly correlated to nitrite concentrations $(n=53, r$ $=0.37, p<0.01)$ but not to nitrate or ammonium.

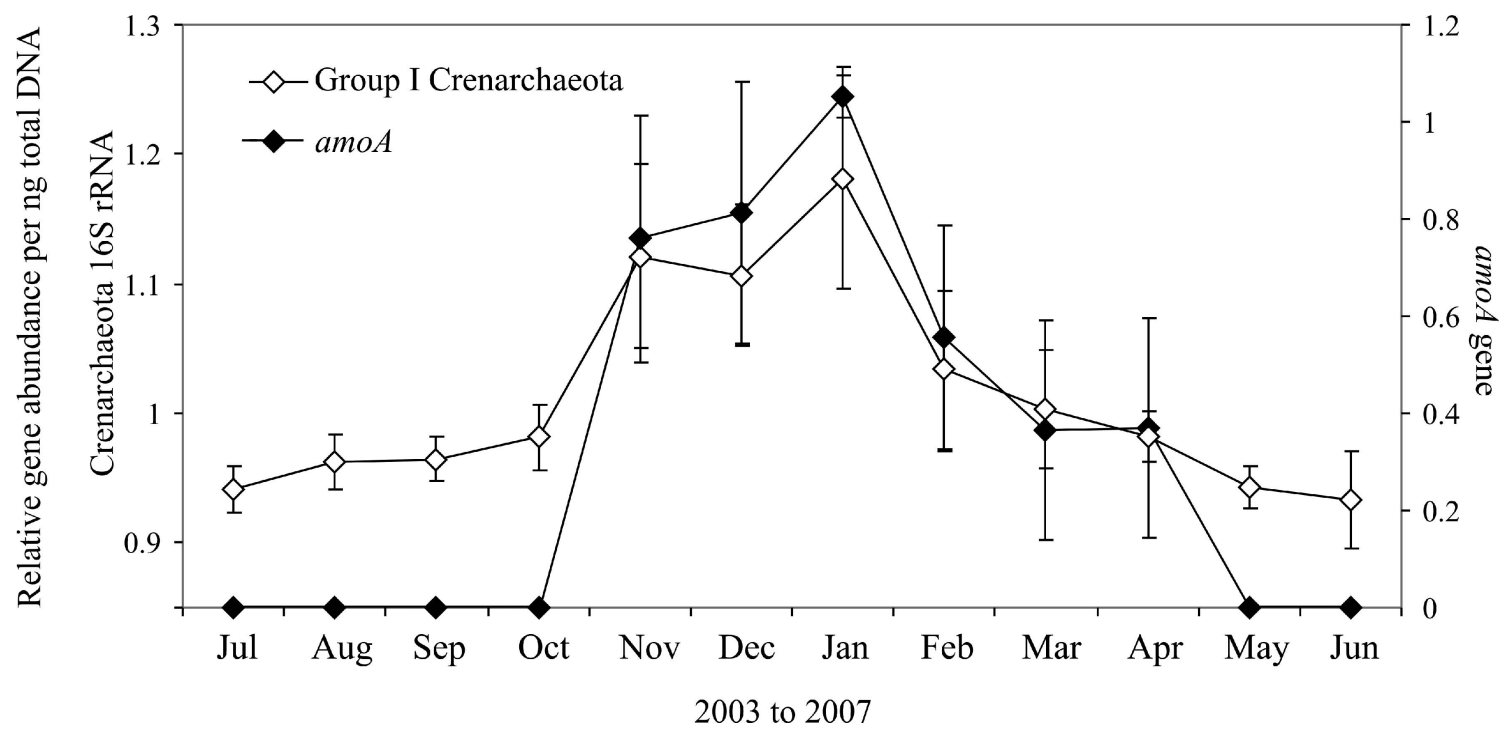

Fig. 3. Average seasonal abundance ( \pm SE) of Group I Crenarchaeota $16 \mathrm{~S}$ rRNA and archaeal amo $A$ genes. Gene copy numbers were quantified by qPCR through the period January 2003-May 2007 at the Blanes Bay Microbial Observatory. Data are normalized to yearly average values $(=1)$. 


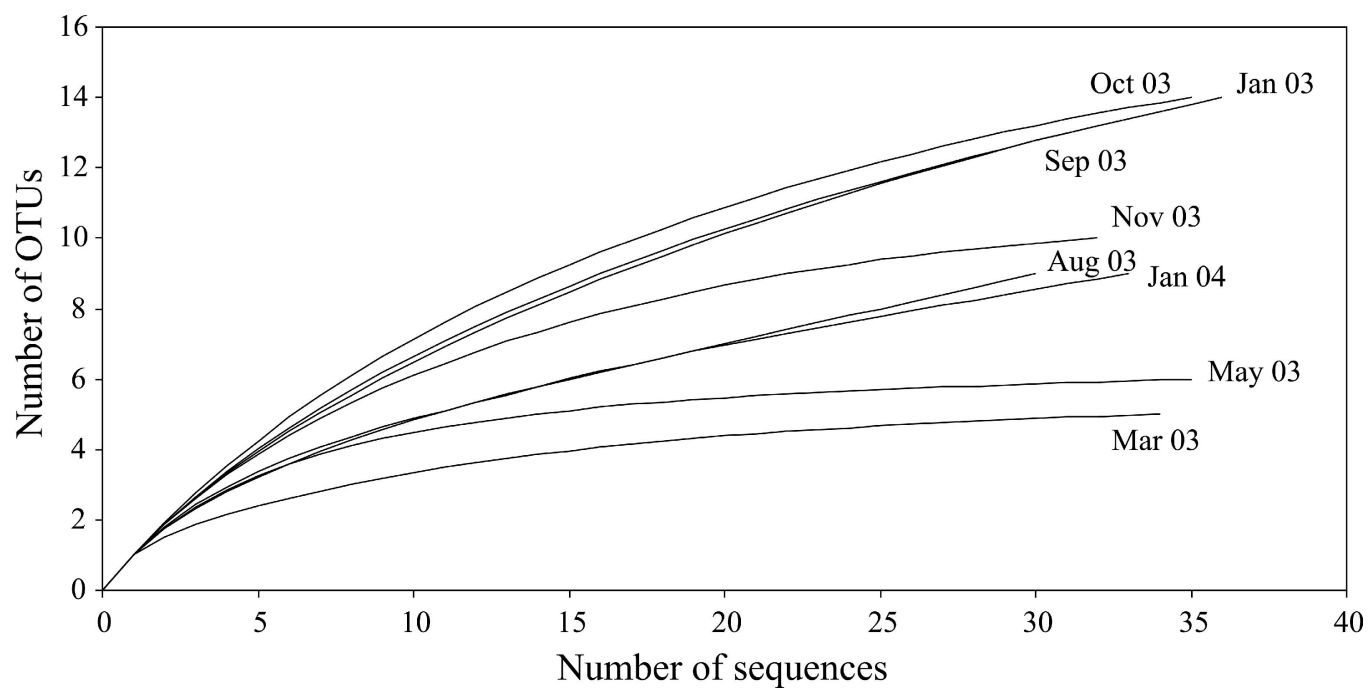

Fig. 4. Rarefaction curves for eight archaeal clone libraries from the Blanes Bay Microbial Observatory. OTUs are defined at a $3 \%$ difference cut off between 16S rRNA gene fragments.

Additionally, we surveyed the relative abundances of the Archaea-specific ammonia monooxygenase (amo A) gene by qPCR. The amo $A$ gene was consistently detected between November and April throughout the complete 4.5-yr study. During the remaining months, it was always below detection limits. The seasonal dynamics of the amo $A$ genes closely followed the abundance of Group I Crenarchaeota (Fig. 3; $n=53, r=0.47, p<0.001$ ) and its relative abundance was positively correlated to nitrate concentrations $(n=53, r=0.38, p<0.01)$.

Seasonal dynamics of the archaeal phylotypes-We constructed eight clone libraries and obtained 246 16S
rRNA gene sequences covering an entire year (Jan 03 to Jan 04) to investigate the seasonal archaeal dynamics in detail. Rarefaction curves showed that some communities were still under-sampled but the abundant groups were probably well-covered at a $97 \%$ sequence identity cut off (Fig. 4). Most of the archaeal sequences belonged to Group II.a Euryarchaeota (43\% of the sequences), II.b Euryarchaeota $(31 \%)$, and Group I Crenarchaeota (18\%). A few (< $10 \%$ ) were from the Euryarchaeota Group III, the freshwater LDS and RC-V clusters (Grosskopf et al. 1998; Glissman et al. 2004), Halobacteriaceae, and Methanospirillaceae (Fig. 5). The phylogenetic trees are given in the Web Appendix.

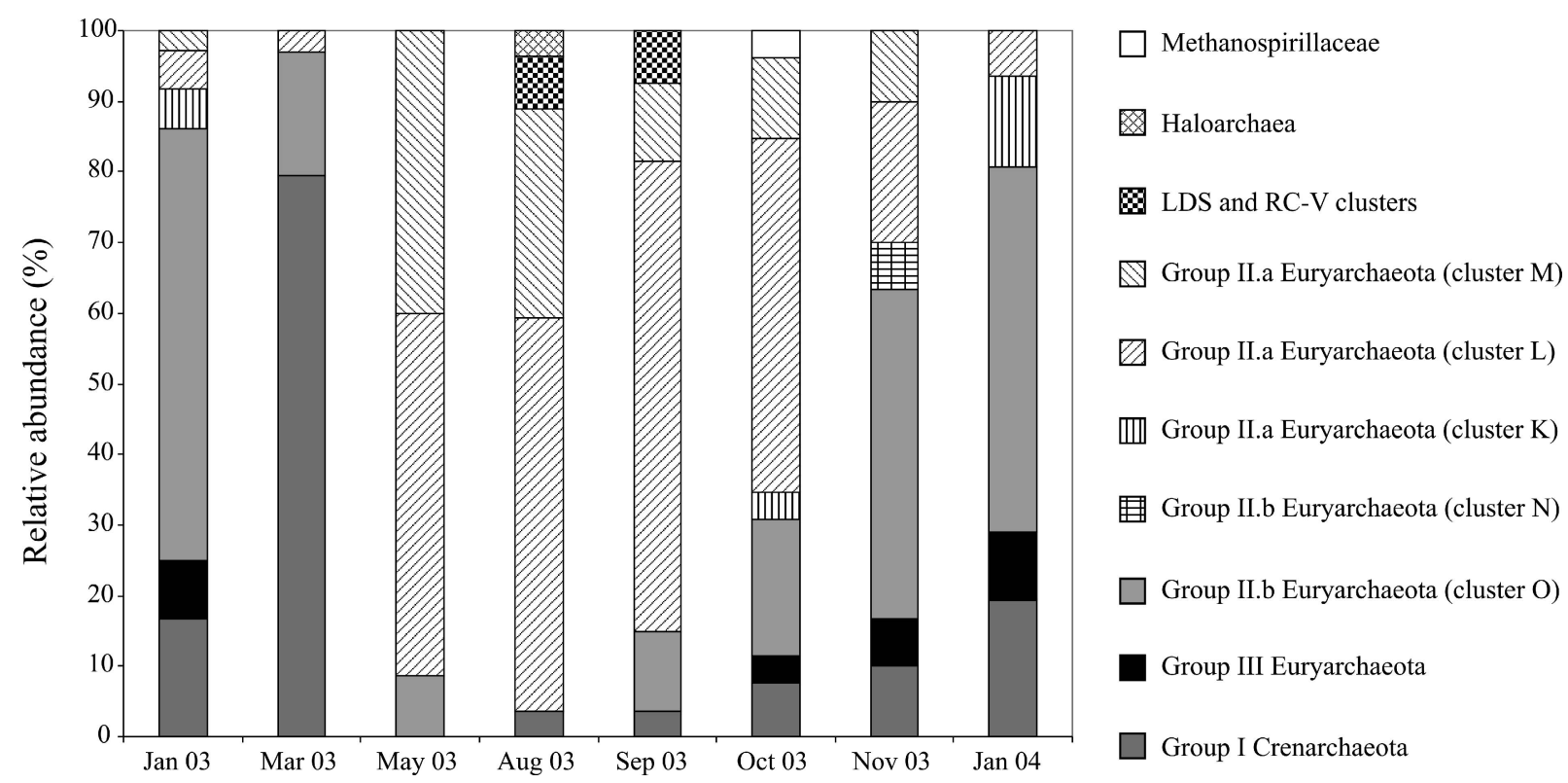

Fig. 5. Seasonal composition of eight archaeal clone libraries, covering an entire year at the Blanes Bay Microbial Observatory in the northwest coastal Mediterranean Sea. Naming of the archaeal clusters K to O follows our own definition given in the Web Appendix. 
Group II euryarchaeal sequences were the most abundant all year round, except in March, and showed consistent seasonal changes in their relative abundances with marked differences between summer and winter (Fig. 5). Group II.b Euryarchaeota dominated the winter libraries, with the highest relative abundance in January (up to $60 \%$; Fig. 5), and separated into two clusters ( $\mathrm{N}$ and O) at a $90 \%$ similarity (see Web Appendix). Cluster O was predominant during late autumn and winter and contained mostly sequences belonging to the WHARN cluster (see Web Appendix). The WHARN cluster corresponds to phylotypes II-CC widely distributed in surface waters of various oceanic provinces (Massana et al. 2000). Cluster N only appeared anecdotally.

Group II.a was the most abundant archaeal type in the clone libraries from May to October and separated into three main clusters (K, L, M, with 89-93\% similarity; see Web Appendix). Most sequences belonged to cluster L, followed by cluster $\mathrm{M}$ mostly detected in May-August (Fig. 5). The reverse Euryarchaeota qPCR primer had one mismatch to members of the cluster L (see Methods) and qPCR results may have underestimated the presence of cluster L and, thus, of Euryarchaeota generally during summer.

Crenarchaeotal sequences were more abundant during winter and all of them belonged to Group I, separated into two clusters at $>93 \%$ similarity (clusters A and B; see Web Appendix). Most of the sequences were closely related to Nitrosopumilus maritimus and there were no seasonal differences in the distributions of the two Group I clusters.

Less abundant groups included Group III Euryarchaeota (see Web Appendix), present during winter and not detected between March and September (Fig. 5), one sequence from Methanospirillaceae, one Halobacteriaceae, and four sequences from the euryarchaeal LDS and RC-V clusters (see Web Appendix), all found in summer and autumn (Fig. 5).

\section{Discussion}

Our long-term study of archaeal dynamics in the coastal northwest Mediterranean Sea revealed that archaea had a marked seasonal periodicity with recurrent peaks of relative abundance in December and January and lower occurrence during summer that matched changes in archaeal community composition. In winter, communities were dominated by Group II.b Euryarchaeota, whereas in summer Group II.a Euryarchaeota were more abundant. To the best of our knowledge, it is the first time that such a seasonal variation within Group II Euryarchaeota was observed. It suggests a probable niche partitioning between the two archaeal types, one developing in winter while the other is present during summer and in low numbers. There are currently no cultivated representatives of marine planktonic Euryarchaeota and their metabolic capabilities are not known. The discovery of proteorhodopsin in some genome fragments related to Group II.b suggests, however, that some may use light to gain a competitive advantage (Frigaard et al. 2006). It may explain why Group II.b Euryarchaeota, and particularly the ubiquitous WHARN cluster, is more abundant in surface waters and declines below the photic zone in many oceanic areas (Massana et al. 1997). Group II.b in Blanes Bay followed patterns of abundance similar to the winter phytoplankton such as the cyanobacteria Prochlorochoccus (Alonso-Sáez et al. 2007), the flagellate Micromonas (Foulon et al. 2008), and diatoms (Agustí and Duarte 2000). We, therefore, hypothesize that members of Group II.b Euryarchaeota, and more specifically members of the WHARN cluster, may be able to use light as an additional energy source, and start growing as soon as nutrient concentrations increase after water column mixing, in correspondence to the first peak of chlorophyll. Euryarchaeota tend, however, to disappear when the main late-winter phytoplankton bloom occurs and may at that time be out-competed by true phototrophs. Conversely, the presence in summer of Group II.a Euryarchaeota matched high-summer phytoplankton lysis rates (Agustí and Duarte 2000). Together with the occurrence of Group II.a in particle-rich coastal waters (Galand et al. 2008), the available data make us speculate that members of the Group II.a, and especially those grouping under cluster L, could have a heterotrophic metabolism possibly closely associated with the summer phytoplankton lysis. Future population-specific studies will help us understand the physiology and ecology of Group II.a and II.b. The different seasonal cycles shown here and the association with major ecosystem processes, such as phytoplankton blooms and lysis, already suggest that they may play separate important roles in the ocean.

The cyclic seasonal dynamics of archaea were different from that of total heterotrophic prokaryotes. In Blanes Bay, heterotrophic prokaryotes were mostly bacteria (Alonso-Sáez et al. 2007), which suggests different responses of bacteria and archaea to environmental controls. During late winter, bacterial abundance increased according to Chl $a$ concentrations, following phytoplankton growth. The late-winter phytoplankton bloom is triggered by the diffusion of nutrient-rich bottom water up to the surface when cold winter temperatures and strong northerly winds break the summer stratification (Duarte et al. 1999). In Blanes Bay, surface nutrient concentrations could also increase following strong rainy events associated with important run off (Guadayol et al. 2009). This late-winter peak of bacteria was characterized by the presence of the alphaproteobacterium Roseobacter (Alonso-Sáez et al. 2007). In turn, the second peak of bacterial abundance in August corresponded to a community dominated by SAR11 (Alonso-Sáez et al. 2007) that appeared at a time when the water column was stratified and nutrients depleted in the upper mixed layer (Duarte et al. 1999). This strong seasonality of both abiotic and biotic factors in the NW Mediterranean probably also influences archaeal dynamics. In particular, the time of summer stratification appears unfavorable for archaea in surface waters, as recently observed in the eastern Mediterranean Sea (Winter et al. 2009).

Euryarchaeota were always the dominant archaeal group in the clone libraries, even during the winter, with the exception of the month of March. This finding confirms preliminary results obtained by fluorescence in situ 
hybridization showing that Crenarchaeota were always rare in Blanes Bay, whereas Euryarchaeota represented up to $6 \%$ of prokaryotes during winter (Alonso-Sáez et al. 2007). This winter predominance of Euryarchaeota contrasts with results showing Crenarchaeota dominating surface winter communities in the North Sea (Wuchter et al. 2006; Herfort et al. 2007) and the Southern Ocean (Murray et al. 1998).

Group I Crenarchaeota in Blanes Bay had, nevertheless, a seasonal periodicity illustrated by higher relative abundance during winter, with a peak in January. Group I is thought to play an important role in the ocean nitrogen cycle by oxidizing ammonia to nitrite (Francis et al. 2005; Wuchter et al. 2006). In Blanes Bay, winter Group I sequences were closely related to the only cultivated planktonic marine archaeum (i.e., Nitrosopumilus maritimus; Könneke et al. 2005). N. maritimus is an autotrophic ammonia-oxidizer that produces nitrite, suggesting that the Group I Crenarchaeota detected in the Blanes Bay also have the potential to oxidize ammonia. The strong relation between the dynamics of the amo $A$ genes (involved in ammonia-oxidation) and Group I, as well as the correlation between the abundance of Group I and nitrite in the water, are additional clues indicating the presence of crenarchaeal nitrifiers in the coastal northwest Mediterranean. Even though Crenarchaeota were not abundant, their activity may be seasonally important. Microbes occurring in low numbers can be functionally important, as shown earlier for nitrogen-fixing bacteria (Montoya et al. 2004) or hydrocarbon-degrading organisms (Teira et al. 2007). The strong peak of nitrite appearing during early winter could, thus, indicate that the Crenarchaeota present at that time of the year are actively oxidizing ammonia to nitrite. However, because phytoplankton can also release nitrite (Lomas and Lipschultz 2006), the exact origin of the winter nitrite in Blanes Bay can not be conclusively determined. Crenarchaeota peaked in January when levels of Chl $a$ decreased after the early winter December peak and before the late-winter March peak. Earlier studies have reported a negative correlation between Chl $a$ and Crenarchaeota in marine and freshwater sites (Murray et al. 1998; Herfort et al. 2007; Auguet and Casamayor 2008), suggesting that organic material excreted by phototrophic primary producers may limit the abundance of Crenarchaeota in the environment (Könneke et al. 2005) or that competition for ammonium may be unfavorable to Crenarchaeota. The low abundance of Crenarchaeota in surface winter waters of the northwest Mediterranean could, thus, be explained by the recurrent late-winter phytoplankton bloom characterizing such regions (Duarte et al. 1999).

Group I Crenarchaeota showed recurrent seasonal patterns over the course of the study with higher relative abundance in winter as unveiled by the qPCR approach. Clone libraries also showed more Group I Crenarchaeota during winter and this good agreement between methods may support the fact that the seasonal patterns observed for the different groups of Euryarchaeota with clone libraries during 2003 are indeed reoccurring and predictable year after year. Such recurrent patterns have been observed earlier for bacteria and may reflect a low degree of functional redundancy in the community, where only a very limited repertoire of species would fill a particular niche (Fuhrman et al. 2006). Similarly, the predictable patterns we observed for Crenarchaeota and Euryarchaeota suggest that the physiology and ecology within each group were controlled by different abiotic or biotic constraints (Auguet et al. 2010).

In summary we showed that archaea had a marked seasonal dynamics in the coastal Mediterranean Sea and that the different components of the community were recurrent in a predictable way. Group II Euryarchaeota was the dominant phyla in the clone libraries but with distinct intra-group seasonal patterns. Group II.b was more abundant during winter, together with putative ammoniaoxidizing Crenarchaeota, while Group II.a was predominant in summer. Thus, the cyclic dynamics of archaea in surface waters of Blanes Bay appear to be closely associated with the strong and predictable seasonality of the Mediterranean Sea.

\section{Acknowledgments}

We thank V. Balague for sampling the deoxyribonucleic acid (DNA) and maintenance of the DNA collection. PEG was supported by a European Marie Curie grant (CRENARC MEIFCT-2007-040247), and CGP by CONSOLIDER-INGENIO 2010 project GRACCIE CSD2007-00067 from the Spanish Ministerio de Ciencia e Innovación (MICINN). Sampling was funded by various projects: European Union project BASICS (EVK3-CT2002-00078), and Spanish grants ESTRAMAR (CTM2004-12631/ MAR, MEC), MODIVUS (CTM2005-04795/MAR), and SUMMER (CTM2008-03309/MAR). Lab research was founded by grants CRENARC and MICINN grant CRENYC CGL200612058-BOS to EOC. We also thank the anonymous reviewers for helpful comments and suggestions.

\section{References}

Agogue, H., M. Brink, J. Dinasquet, and G. J. Herndl. 2008. Major gradients in putatively nitrifying and non-nitrifying Archaea in the deep North Atlantic. Nature 456: 788-791, doi:10.1038/nature07535

Agustí, S., And C. M. Duarte. 2000. Strong seasonality in phytoplankton cell lysis in the NW Mediterranean littoral. Limnol. Oceanogr. 45: 940-947, doi:10.4319/ 10.2000.45.4.0940

Alonso-SÁez, L., AND others. 2007. Seasonality in bacterial diversity in north-west Mediterranean coastal waters: Assessment through clone libraries, fingerprinting and FISH. FEMS Microbiol. Ecol. 60: 98-112, doi:10.1111/j. 1574-6941.2006.00276.x

, AND - 2008. Factors controlling the year-round variability in carbon flux through bacteria in a coastal marine system. Ecosystems 11: 397-409, doi:10.1007/ s10021-008-9129-0

Ashelford, K. E., A. J. Weightman, and J. C. Fry. 2002. PRIMROSE: A computer program for generating and estimating the phylogenetic range of $16 \mathrm{~S}$ rRNA oligonucleotide probes and primers in conjunction with the RDP-II database. Nucleic Acids Res. 30: 3481-3489, doi:10.1093/nar/ gkf450

Auguet, J.-C., A. Barberan, and E. O. Casamayor. 2010. Global ecological patterns in uncultured Archaea. ISME J. 4: 182-190, doi:10.1038/ismej.2009.109

-, And E. O. Casamayor. 2008. A hotspot for cold crenarchaeota in the neuston of high mountain lakes. 
Environ. Microbiol. 10: 1080-1086, doi:10.1111/j. 1462-2920.2007.01498.x

Duarte, C. M., S. Agustí, H. Kennedy, and D. Vaqué. 1999. The Mediterranean climate as a template for Mediterranean marine ecosystems: The example of the northeast Spanish littoral. Prog. Oceanogr. 44: 245-270, doi:10.1016/ S0079-6611(99)00028-2

EdgAR, R. C. 2004. MUSCLE: A multiple sequence alignment method with reduced time and space complexity. BMC Bioinformatics 5: 113, doi:10.1186/1471-2105-5-113

Felsenstein, J. 2008. PHYLIP (Phylogeny Inference Package) version 3.68. Distributed by the author. Department of Genome Sciences, Univ. of Washington.

Foulon, E., F. Not, F. Jalabert, T. Cariou, R. Massana, and N. SimON. 2008. Ecological niche partitioning in the picoplanktonic green alga Micromonas pusilla: Evidence from environmental surveys using phylogenetic probes. Environ. Microbiol. 10: 2433-2443, doi:10.1111/j.1462-2920.2008.01673.x

Francis, C. A., K. J. Roberts, J. M. Beman, A. E. SAntoro, And B. B. OAKLEY. 2005. Ubiquity and diversity of ammoniaoxidizing archaea in water columns and sediments of the ocean. Proc. Natl. Acad. Sci. U.S.A. 102: 14683-14688, doi:10.1073/pnas.0506625102

Frigaard, N. U., A. Martinez, T. J. Mincer, and E. F. Delong. 2006. Proteorhodopsin lateral gene transfer between marine planktonic Bacteria and Archaea. Nature 439: 847-850, doi:10.1038/nature04435

Fuhrman, J. A., I. Hewson, M. S. Schwalbach, J. A. Steele, M. V. Brown, AND S. NAeEm. 2006. Annually reoccurring bacterial communities are predictable from ocean conditions. Proc. Natl. Acad. Sci. U.S.A. 103: 13104-13109, doi:10.1073/ pnas.0602399103

Galand, P. E., E. O. Casamayor, D. L. Kirchman, M. Potvin, AND C. Lovejoy. 2009a. Unique archaeal assemblages in the Arctic Ocean unveiled by massively parallel tag sequencing. ISME J. 3: 860-869, doi:10.1038/ismej.2009.23

, C. Lovejoy, A. K. Hamilton, R. G. Ingram, E. Pedneault, and E. Carmack. 2009b. Archaeal diversity and a gene for ammonia oxidation are coupled to oceanic circulation. Environ. Microbiol. 11: 971-980, doi:10.1111/ j.1462-2920.2008.01822.x

J. Pouliot, and W. F. Vincent. 2008. Heterogeneous archaeal communities in the particle-rich environment of an arctic shelf ecosystem. J. Marine Syst. 74: 774-782, doi:10.1016/j.jmarsys.2007.12.001

Garcia-Martinez, J., and F. Rodriguez-Valera. 2000. Microdiversity of uncultured marine prokaryotes: The SAR11 cluster and the marine Archaea of Group I. Mol. Ecol. 9: 935-948, doi:10.1046/j.1365-294x.2000.00953.x

Glissman, K., K.-J. Chin, P. Casper, and R. Conrad. 2004. Methanogenic pathway and archaeal community structure in the sediment of eutrophic lake Dagow: Effect of temperature. Microb. Ecol. 48: 389-399, doi:10.1007/s00248-003-2027-2

Grosskopf, R., S. Stubner, and W. Liesack. 1998. Novel euryarchaeotal lineages detected on rice roots and in the anoxic bulk soil of flooded rice microcosms. Appl. Environ. Microbiol. 64: 4983-4989.

Guadayol, O., And others. 2009. Episodic meteorological and nutrient-load events as drivers of coastal planktonic ecosystem dynamics: A time-series analysis. Mar. Ecol. Prog. Ser. 381: 139-155, doi:10.3354/meps07939

Hammer, Ø., D. A. T. HARPER, AND P. D. Ryan. 2001. PAST: Paleontological statistics software package for education and data analysis. Paleontol. Electro. 4: 9.

Hansman, R. L., S. Griffin, J. Watson, E. R. M. Druffel, A. E. Ingalls, AND L. I. Aluwihare. 2009. The radiocarbon signature of microorganisms in the mesopelagic ocean. Proc. Natl. Acad. Sci. U.S.A. 106: 6513-6518, doi:10.1073/ pnas.0810871106

HERFort, L., AND OTHERS. 2007. Variations in spatial and temporal distribution of Archaea in the North Sea in relation to environmental variables. FEMS Microbiol. Ecol. 62: 242-257, doi:10.1111/j.1574-6941.2007.00397.x

Ingalls, A. E., S. R. Shah, R. L. Hansman, L. I. Aluwihare, G. M. Santos, And E. R. M. Druffel. 2006. Quantifying archaeal community autotrophy in the mesopelagic ocean using natural radiocarbon. Proc. Natl. Acad. Sci. U.S.A. 103: 6442-6447, doi:10.1073/pnas.0510157103

Karner, M. B., E. F. Delong, and D. M. Karl. 2001. Archaeal dominance in the mesopelagic zone of the Pacific Ocean. Nature 409: 507-510, doi:10.1038/35054051

Könneke, M., A. E. Bernhard, J. R. De La Torre, C. B. Walker, J. B. Waterbury, and D. A. Stahl. 2005. Isolation of an autotrophic ammonia-oxidizing marine archaeon. Nature 437: 543-546, doi:10.1038/nature03911

Lomas, M. W., And F. Lipschultz. 2006. Forming the primary nitrite maximum: Nitrifiers or phytoplankton? Limnol. Oceanogr. 51: 2453-2467, doi:10.4319/1o.2006.51.5.2453

Martin-Cuadrado, A. B., and others. 2008. Hindsight in the relative abundance, metabolic potential and genome dynamics of uncultivated marine archaea from comparative metagenomic analyses of bathypelagic plankton of different oceanic regions. ISME J 2: 865-886, doi:10.1038/ ismej. 2008.40

Massana, R., E. F. Delong, C. Pedros-Alio, A. E. Murray, and C. M. Preston. 2000. A few cosmopolitan phylotypes dominate planktonic archaeal assemblages in widely different oceanic provinces. Appl. Environ. Microbiol. 66: 1777-1787, doi:10.1128/AEM.66.5.1777-1787.2000

, A. E. Murray, C. M. Preston, and E. F. Delong. 1997. Vertical distribution and phylogenetic characterization of marine planktonic Archaea in the Santa Barbara Channel. Appl. Environ. Microbiol. 63: 50-56.

Montoya, J. P., C. M. Holl, J. P. Zehr, A. Hansen, T. A. Villareal, and D. G. Capone. 2004. High rates of $\mathrm{N}_{2}$ fixation by unicellular diazotrophs in the oligotrophic Pacific Ocean. Nature 430: 1027-1032, doi:10.1038/ nature 02824

Murray, A. E., C. M. Preston, R. Massana, L. T. Taylor, A. Blakis, K. Wu, and E. F. Delong. 1998. Seasonal and spatial variability of bacterial and archaeal assemblages in the coastal waters near Anvers Island, Antarctica. Appl. Environ. Microbiol. 64: 2585-2595.

Ouverney, C. C., And J. A. Fuhrman. 2000. Marine planktonic Archaea take up amino acids. Appl. Environ. Microbiol. 66: 4829-4833, doi:10.1128/AEM.66.11.4829-4833.2000

Pouliot, J., P. E. Galand, C. Lovejoy, and W. F. Vincent. 2009. Vertical structure of archaeal communities and the distribution of ammonia monooxygenase A gene variants in two meromictic High Arctic lakes. Environ. Microbiol. 11: 687-699, doi:10.1111/j.1462-2920.2008.01846.x

Schloss, P. D., And J. Handelsman. 2005. Introducing DOTUR, a computer program for defining operational taxonomic units and estimating species richness. Appl. Environ. Microbiol. 71: 1501-1506, doi:10.1128/AEM.71.3.1501-1506.2005

Sozzi, G., L. Roz, D. Conte, L. Mariani, F. Andriani, P. Verderio, ANd U. PAstorino. 2005. Effects of prolonged storage of whole plasma or isolated plasma DNA on the results of circulating DNA quantification assays. J. Natl. Cancer Inst. 97: 1848-1850, doi:10.1093/jnci/dji432

Teira, E., I. Lekunberri, J. M. Gasol, M. Nieto-Cid, X. A. Alvarez-Salgado, and F. G. Figueiras. 2007. 
Dynamics of the hydrocarbon-degrading Cycloclasticus bacteria during mesocosm-simulated oil spills. Environ. Microbiol. 9: 2551-2562, doi:10.1111/j.1462-2920.2007. 01373.x

, T. Reinthaler, A. Pernthaler, J. Pernthaler, and G. J. HERndL. 2004. Combining catalyzed reporter depositionfluorescence in situ hybridization and microautoradiography to detect substrate utilization by Bacteria and Archaea in the deep ocean. Appl. Environ. Microbiol. 70: 4411-4414, doi:10.1128/AEM.70.7.4411-4414.2004

Winter, C., M. E. Kerros, and M. G. Weinbauer. 2009. Seasonal changes of bacterial and archaeal communities in the dark ocean: Evidence from the Mediterranean Sea. Limnol. Oceanogr. 54: 160-170.

Wuchter, C., AND OTHERs. 2006. Archaeal nitrification in the ocean. Proc. Natl. Acad. Sci. U.S.A. 103: 12317-12322, doi:10.1073/pnas.0600756103

Associate editor: Wade H. Jeffrey

Received: 30 November 2009 Accepted: 10 May 2010 Amended: 24 June 2010 